\title{
In silico discovery of novel transcription factors regulated by mTOR-pathway activities
}

\author{
Agnieszka Jablonska ${ }^{1}$ and Natalia Polouliakh ${ }^{2,3,4}$ * \\ 1 Faculty of Biotechnology and Food Sciences, Lodz University of Technology, Lodz, Poland \\ ${ }^{2}$ Fundamental Research Laboratories, Sony Computer Science Laboratories Inc., Tokyo, Japan \\ ${ }^{3}$ Systems Biology Institute, Tokyo, Japan \\ ${ }^{4}$ Graduate School of Medicine, Yokohama City University, Yokohama, Japan
}

\section{Edited by:}

Firas H. Kobeissy, University of

Florida, USA

Reviewed by:

Thomas Bouckenooghe, University of Lille 2, France

Mohamad Musbah Almedawar, American University of Beirut, Lebanon

\section{*Correspondence:}

Natalia Polouliakh, Fundamental

Research Laboratories, Sony

Computer Science Laboratories Inc.

Takanawa Muse Bldg., 3-14-13

Higashigotanda Shinagawa-ku,

Tokyo 141-0022, Japan

e-mail:nata@csl.sony.co.jp
The mammalian target of rapamycine (mTOR) pathway is a key regulator of cellular growth, development, and ageing, and unraveling its control is essential for understanding life and death of biological organisms. A motif-discovery workbench including nine tools was used to identify transcription factors involved in five basic (Insulin, MAPK, VEGF, Hypoxia, and mTOR core) activities of the mTOR pathway. Discovered transcription factors are classified as "process-specific" or "pathway-ubiquitous" with highlights toward their regulating/regulated activities within the MTOR pathway. Our transcription regulation results will facilitate further research on investigating the control mechanism in mTOR pathway.

Keywords: mTOR pathway, orthologous genes, transcription factors, bioinformatic tools and databases, cell signaling

\section{INTRODUCTION}

Computational analysis of transcription regulation of biological pathways is a challenging task because a single pathway may be interconnected to multiple upstream and downstream pathways and, as a consequence, might be regulated by various groups of transcription factors (Schmelzle and Hall, 2000; Hay and Sonenberg, 2004; Inoki and Guan, 2006; Polouliakh et al., 2006, 2009; Laplante and Sabatini, 2009; Caron et al., 2010). Because the transcriptional level is a fundamental mechanism that is well conserved in all cellular systems, a cross-species comparison approach should increase the confidence of the analysis. A further way to reduce false positives is to combine the consistent part of the results obtained on multiple analytic tools.

In this work, a transcription regulation network of orthologous human-mouse mTOR (mammalian target of rapamycin) pathway genes was investigated by using nine public motifdiscovery tools published in the last decade. TOR was first discovered in yeast (Heitman et al., 1991), then identified as evolutionarily conserved (Brown et al., 1994; Sabatini et al., 1994), and finally functionally characterized in fungi yeast (Lorberg and Hall, 2004), animals (Hall, 2008), and plants (Deprost et al., 2007). It has a high molecular weight and several distinctive domains. In particular, mTOR consists of 2549 amino acids and possesses a kinase domain at the $\mathrm{C}$ terminal, which displays homology to the phosphatidylinositol 3-kinase (PI3K) catalytic domain. PIK-related kinases are responsible for numerous cellular processes, e.g., cell growth and development, DNA-damage detection, and telomere maintenance (Schmelzle and Hall, 2000; Hay and Sonenberg, 2004). Because of its structure, mTOR can create two functional protein complexes with two other proteins: one with MLST8 and RICTOR and one with MLST and
RPTOR. Since it can form such structures, mTOR has to be analyzed in the context of a wider signaling network (Inoki et al., 2005).

An mTOR pathway is an important regulator of embryogenesis, angiogenesis, adipogenesis, autophagy, and cell cycle. It can be divided into several branches responsible for actin and microtubule organization, protein and lipid synthesis, cell growth and cell differentiation, and autophagy (Laplante and Sabatini, 2009). In addition, the involvement of an mTOR pathway in the aging process and pathological changes such as tumor formation, cancer, insulin resistance, and diabetes has been pointed out by many researches (Laplante and Sabatini, 2009; Stanfel et al., 2009; Caron et al., 2010). What's more, disruption of genes from mTOR pathway (e.g., TSC1, TSC2, and TOR) results in early embryonic death both in Drosophilla and mammals, suggesting that TOR plays a crucial role in the development (Kobayashi et al., 2001; Murakami et al., 2004; Inoki et al., 2005). In humans, cell hypertrophy, hyper-function and hyperplasia, typically associated with activation of mTOR, contribute to diseases associated with aging, such as myocardial infarctions and strokes, osteoporosis, cancer, autoimmune disease, arthritis, obesity, diabetes, macula-degeneration, and Alzheimer's and Parkinson's diseases (Blagosklonny, 2006; Johnson et al., 2013; O’Neill and Hardie, 2013).

An mTOR pathway is regulated by various signals. It can be activated by availability of amino acids and nutrients, presence of growth factors and hormones, and it may be halted by the action of such factors as energy stress, hypoxia, and osmotic changes (Inoki et al., 2005; Inoki and Guan, 2006; Caron et al., 2010). It is also regulated by the level of gene expression, which is controlled by transcription factors (TFs) within the pathway. 
When investigating transcription regulation, it is necessary to consider both the comparatively short promoter regions in the proximity of a transcriptional start site (TSS), where the polymerase II transcription initiation complex forms, and distantly located enhancer regions that may interact with one or more promoter sites of neighboring genes (Serizawa et al., 2003) and whose distance from the TSS can exceed $100 \mathrm{~kb}$.

Given that scientific tools can be difficult to install, it is particularly helpful for biologists to be able to use these tools through a web-user interface. Our investigations were focused on tools available online and showed that nine out of thirty-two tested online motif discovery programs are easy and suitable for the analysis, while others should be handled offline or require additional data management.

As a result of our study with selected tools, 61 transcription factors for human and 50 transcription factors for mouse were identified, and 24 and 13 transcription factors, respectively, were then selected for the discussion on their activity in mTOR pathway. Twenty-one transcription factors between human and mouse were orthologous, and the 13 most frequent of them are discussed in this paper.

Transcription factors regulating an mTOR pathway and transcription factors regulated by an mTOR pathway are indicated separately. We believe that the created transcription regulation map can extend the knowledge from already verified transcription factors to the new hypothesis of transcription regulation of an mTOR pathway including feedback loops. Our guidance on a bunch of recent motif-discovery tools will be helpful for a large scope of audience and should trigger further discussion on optimization of tools and analysis workflow (Ghosh et al., 2011).

\section{MATERIALS AND METHODS mTOR PATHWAY GENE DATASET}

The mTOR human pathway was downloaded from the KEGG database (map04150) and included 49 human genes with 43 orthologous partners in mouse. Promoter regions of 5000 base pairs upstream and 500 base pairs downstream with their representative transcriptional start sites (TSS) were retrieved from the DBTSS database (Yamashita et al., 2010).

\section{MOTIF DISCOVERY TOOLS AND MOTIF SELECTION}

Thirty-one non-commercial online motif-discovery programs, which are already popular for the analysis of biological data or were newly developed in the last decade, were investigated. In our set of tools we included programs that could analysis orthologous promoters (e.g., Pscan, TransFind), programs with diverse methodologies (e.g., MEME_Chip, TFM-explorer, Pscan) and programs that link the results to the expression data to augment probability likelihood of predicted motifs (e.g., MEME_Chip, PASTAA, TRAP). Next, each of selected program had to fulfill the following four characteristics: (1) capability to handle a large data size $(300 \mathrm{~Kb})$ and return results in less than $24 \mathrm{~h}$; (2) report motif results for each DNA sequence or report motif results across the whole dataset; (3) execute a software operation without manual parameter adjustment; and (4) annotate resulted motifs in currently available databases.

Five main programs, which report identified motifs in each sequence, namely, Pscan (Zambelli et al., 2009), PASTAA (Roider et al., 2009), MEME_Chip (Machanick and Bailey, 2011), TFM-Explorer (Defrance and Touzet, 2006), and XXmotif (Luehr et al., 2012) were selected, and four supportive programs, which report motifs over a whole dataset, namely, Cscan (Zambelli et al., 2012), LocaMo (Vandenbon et al., 2013), TransFind (Kielbasa et al., 2010), and TRAP (Roider et al., 2007), were selected.

All programs were executed in their default parameter setting. Table 1 lists features of the nine programs used for the analysis.

Twenty-two online programs that were investigated and found unsuitable for our analysis are listed as follows: BioProspector (Liu et al., 2001), CompareProspector (Liu et al., 2004), CompMoby (Chaivorapol et al., 2008), ConTra (Broos et al., 2011;) ConSite (Sandelin et al., 2004), Improbizer (Ao et al., 2004), Gibbs Motif Sampler (Thompson et al., 2003), Lasagna (Lee and Huang, 2013), MDScan (Liu et al., 2002), Melina (Poluliakh et al., 2003), MEME (Bailey et al., 2009), Mobyle @Pasteur tfscan (Néron et al., 2009), MotifSearch (Dinh et al., 2011), MotifViz (Fu et al., 2004), MotifVoter (Wijaya et al., 2008), PhyloGibbs (Siddharthan et al., 2005), RSAT consensus, rVista (Loots and Ovcharenko, 2004), SeSiMCMC (Favorov et al., 2005), SCOPE (Carlson et al., 2007), WebMOTIFS (Romer et al., 2007), and YMF (Sinha and Tompa, 2003).

Initial motifs were collected in accordance with two conditions: first, each of the five main selected programs should identify a motif and, second, some supportive programs should also find the same motif. Details of collected motifs are represented in Supporting Figure 1, together with their target genes and software programs used.

To find the most co-regulated genes within the dataset, the average number of transcription factors per gene was calculated by dividing the total number of TFBS motifs found by PScan, PASTAA, MEME_Chip, TFM-Explorer, and XXmotif in a human dataset $(1,295)$, by the total number of genes in the human dataset (49) that resulted in $26.43(S D \pm 7.87)$ transcription-factor motifs per gene, shown above the red horizontal line in Supporting Figure 1A, hereafter called a gene threshold (Gene_TH). This resulted in 24 genes, possessing more than 26 predicted motifs in their promoters for human, including their multiple occurrences.

The top 24 out of 61 (40\%) human transcription factors found in the promoters of genes above the gene threshold were taken and denoted as those above a transcription factor threshold (TF_TH). This selection showed that at least half of the 24 genes possessed each of the 24 above-selected transcription-factor motifs in their promoters. The top-occurring transcription factors was SP1 with its highest frequency and top occurrence of seven copies in the RPS6KA3 gene, and the last-occurring transcription factor was ARNT found in 12 genes with single occurrence (Supporting Figure 1A). This 24-by-24 matrix is named an intersection zone.

The intersection zone was used to produce an mTOR map by CellDesigner4.3 (Funahashi et al., 2003) with those TFs having multiple copies in the promoters or those TFs already mentioned in the literature in relation to mTOR (Figure 1). The transcription-regulation map was divided into an $m$ TOR core and ten modules for convenience to point out to the transcription factors involved in different activities of the mTOR pathway. Modules were then classified into pre-core transcriptional 


\begin{tabular}{|c|c|c|c|c|c|c|c|c|c|c|}
\hline 蹄 & 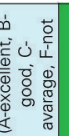 & $\varangle$ & $\infty$ & $\varangle$ & $\varangle$ & $\varangle$ & $\ll$ & $<$ & $\varangle$ & $\infty$ \\
\hline : & 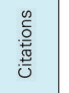 & ஜ & $\overline{5}$ & 产 & $\approx$ & 0 & $=$ & N & $=$ & $\stackrel{\circ}{\circ}$ \\
\hline$\frac{\square}{\dddot{\varpi}}$ & 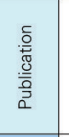 & 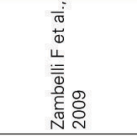 & 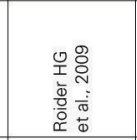 & 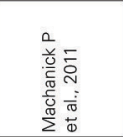 & 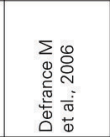 & 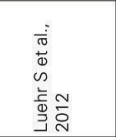 & 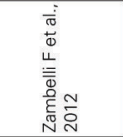 & 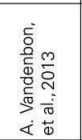 & 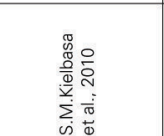 & \\
\hline 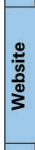 & & 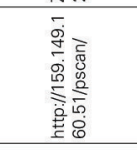 & 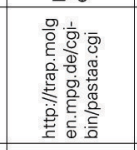 & 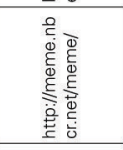 & 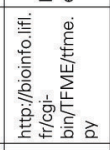 & 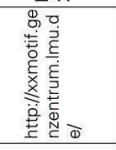 & 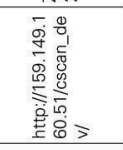 & 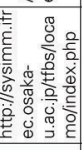 & 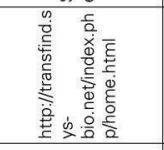 & 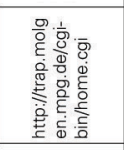 \\
\hline 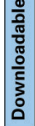 & $\sum_{\substack{\infty \\
\stackrel{D}{\infty}}}^{0}$ & $\stackrel{\mathscr{ల}}{\rightleftharpoons}$ & $\stackrel{\mathscr{D}}{\rightleftharpoons}$ & $\stackrel{\circ}{z}$ & $\stackrel{\mathscr{D}}{\rightleftharpoons}$ & $\stackrel{\infty}{\rightleftharpoons}$ & $\stackrel{\circ}{2}$ & $\stackrel{\circ}{2}$ & $\stackrel{\circ}{2}$ & $\stackrel{\mathscr{g}}{=}$ \\
\hline 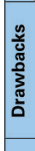 & & 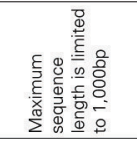 & 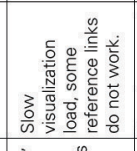 & 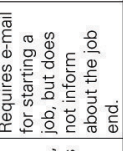 & 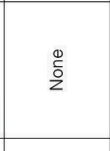 & $\begin{array}{l}\frac{0}{0} \\
\frac{0}{\delta}\end{array}$ & 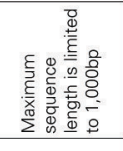 & 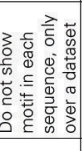 & 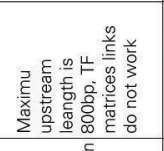 & 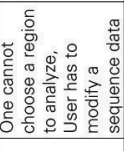 \\
\hline 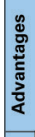 & & 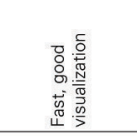 & 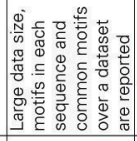 & 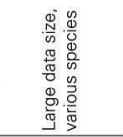 & 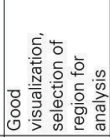 & 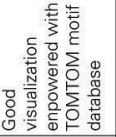 & 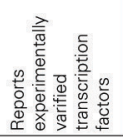 & 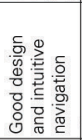 & 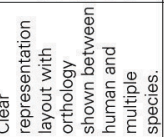 & 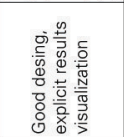 \\
\hline 竞 & 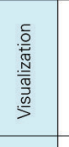 & 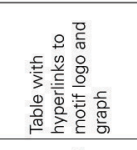 & $\frac{\frac{0}{0}}{\frac{0}{\pi}}$ & 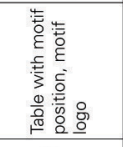 & 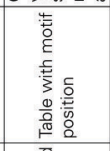 & 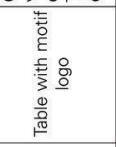 & $\frac{\frac{0}{00}}{\frac{0}{00}}$ & 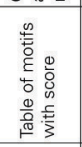 & 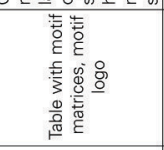 & 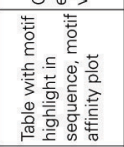 \\
\hline के & 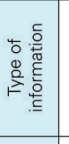 & 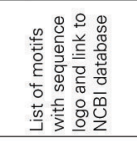 & 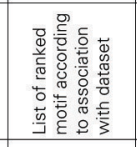 & 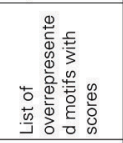 & 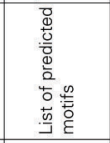 & 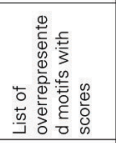 & 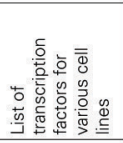 & 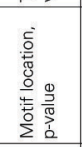 & 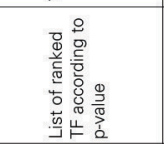 & 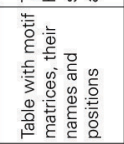 \\
\hline & 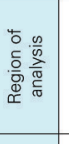 & 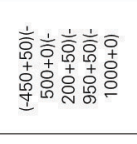 & 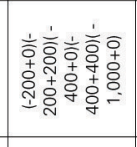 & 辌 & 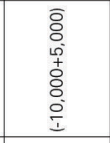 & 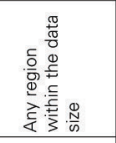 & 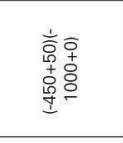 & 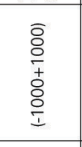 & 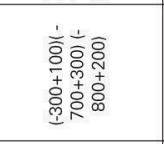 & 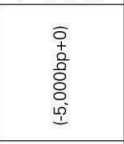 \\
\hline $\mid \begin{array}{l}\underline{\underline{z}} \\
\underline{\underline{\underline{z}}}\end{array}$ & 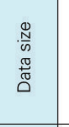 & 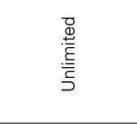 & 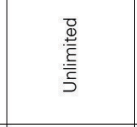 & $\frac{\text { 营 }}{2}$ & 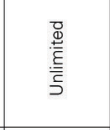 & 商 & 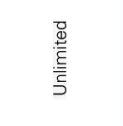 & 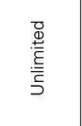 & 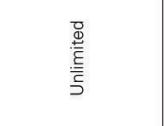 & 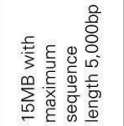 \\
\hline & 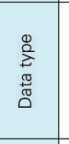 & $\stackrel{\varrho}{\varrho}$ & 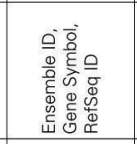 & 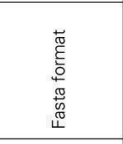 & 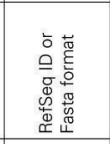 & 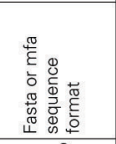 & 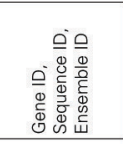 & 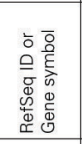 & $\begin{array}{l}\stackrel{\varrho}{0} \\
\stackrel{0}{0} \\
0 \\
\end{array}$ & 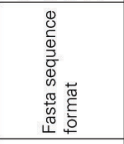 \\
\hline & 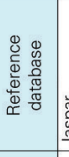 & 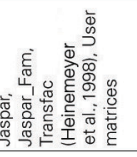 & 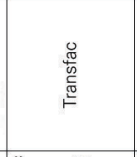 & 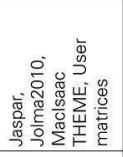 & 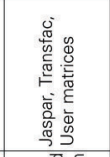 & 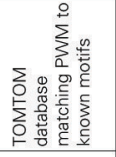 & 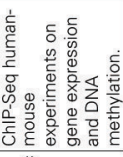 & 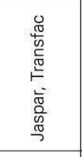 & 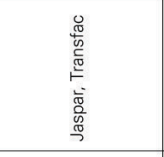 & 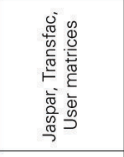 \\
\hline$\frac{\bar{f}}{\overline{\mathbf{v}}}$ & 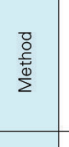 & 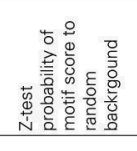 & 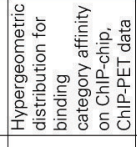 & 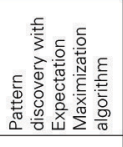 & 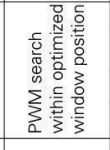 & 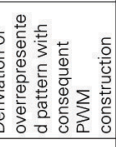 & 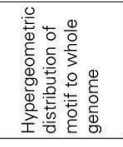 & 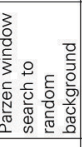 & 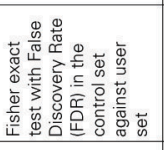 & 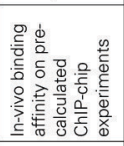 \\
\hline & 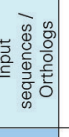 & 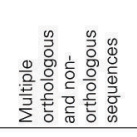 & 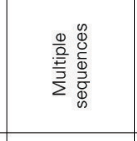 & 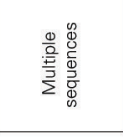 & 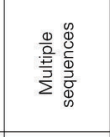 & 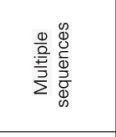 & 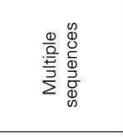 & 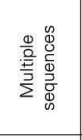 & 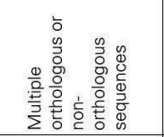 & 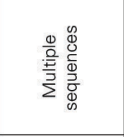 \\
\hline 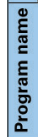 & & 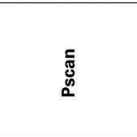 & 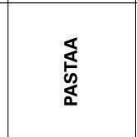 & 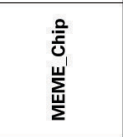 & 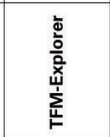 & 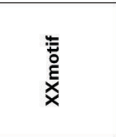 & 藨 & 遌 & 莺 & 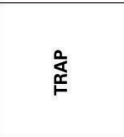 \\
\hline & & & & 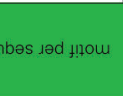 & & & & & 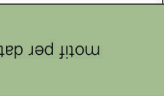 & \\
\hline
\end{tabular}

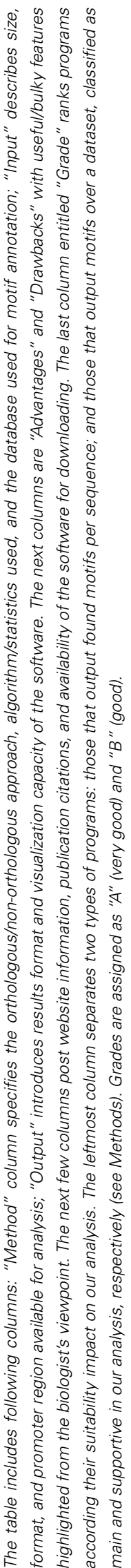




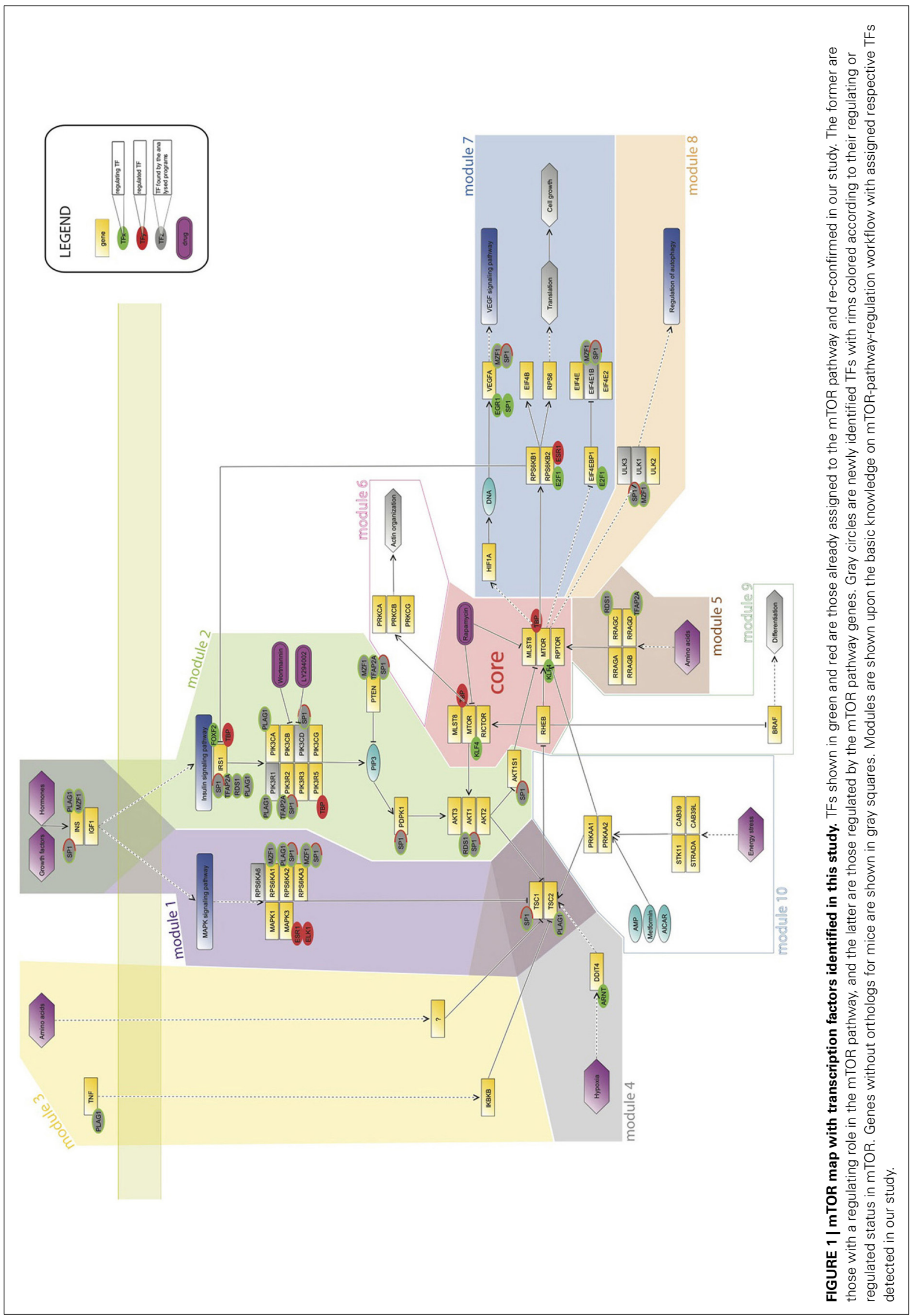


modules and post-core transcriptional modules according to their place within the pathway.

Transcription factors were classified as those that regulate the mTOR pathway and those that are regulated by the mTOR pathway, representing them in green and/or red circles on the map in Figure 1. Newly identified TFs are shown in gray circles with a respective color rim depending on their role in the mTOR pathway. Human genes without orthologs in mice are shown in gray squares on the map (Figure 1). The intersection zone for human is extended to mouse data to see commonalities between the two species (Supporting Figures 1A,B).

\section{RESULTS}

\section{mTOR-PATHWAY TRANSCRIPTION FACTORS}

Transcription factors (TF) and genes allocated in the intersection zone (see Methods) were selected as candidates for regulating an mTOR pathway. The initial identified number of TFs was 61 for human and 50 for mouse with 21 orthologs (Figure 1 and Supporting Figure 1). Nine transcription factors identified in our study were already assigned to the activities of an mTOR pathway, but their target genes were not specified. Those TFs are following: SP1, KLF4, TBP, ELK1, ESR1, E2F1, FOXF2, EGR1, and ARNT, shown with respective green or red colors in Figure 1 according to their regulating or regulated role for in regard to an mTOR pathway. TFs having orthologous binding motifs in mouse are marked in Supporting Figure 1C with asterisks. Previously unknown transcription factors identified in our study in mTORpathway genes are shown in gray on the map in Figure 1. We found particularly interesting those TFs with number of binding sites exceeding three occurrences per gene, i.e., high-affinity TFs, and those appearing in more than a half of the promoters of genes in the dataset, i.e., in our intersection zone (see Methods). Activities of various modules with assigned transcription factors are discussed in the next sub-sections.

\section{mTOR CORE, PRE-CORE, POST-CORE TRANSCRIPTIONAL MODULES}

The pre-core mTOR modules are listed as following: module 1 -induced by growth factors and hormones possessing MAPK cascade and RPS6K genes; module 2-activated by growth factors and hormones and involved in insulin signaling pathway associated with PIK3 genes and AKT genes; module 3-regulated by extracellular amino acids and TNF; module 4-possessing hypoxia dependent genes; module 5-connected with the balance of amino acids inside the cell and related to the RRAG gene group; module 9-linked with cell differentiation; module 10containing energy-stress-related genes such as CAB3, STRADA, and STK11 as well as genes influenced by drug administration.

Post-core transcriptional modules are listed as follows: module 6-associated with actin organization; module 7-connected to VEGF pathway conducting cell development and growth; and module 8-modulating autophagy. As an mTOR pathway core, an RHEB gene and two blocks of genes with MTOR-RICTOR and MTOR-RPTOR interacting pairs, respectively, is considered. Modules 3, 6, 8, and 9 are shown on the map in Figure 1, but they are not discussed in this paper because no reliable motifs were identified in our study.

\section{MODULE ONE OF MAPK-SIGNALING PATHWAY AND MODULE TWO OF INSULIN-SIGNALING PATHWAY}

In module 1, which embodies MAPK signaling pathway, five transcription factors were identified. Two of them, ESR1 and ELK1, are already known, and three newly identified in our study are SP1, MZF1 (myeloid zinc finger 1), and PLAG1 (Pleiomorphic adenoma gene 1 protein).

Both ELK1 (an ETS domain-containing protein) and ESR1 (a nuclear steroid receptor) interact with the MAPK3 gene (Polouliakh et al., 2006). ELK1 is a nuclear target for a MAPK cascade (Uht et al., 2007). ESR1 is a homodimer composed of several domains crucial for hormone binding and form heterodimers with ESR2 (estrogen receptor 2). In the mTOR pathway, ESR1 is inhibited by phosphophorylation regulated by RPS6KB1 (S6K1) from a VEGF signaling pathway and a MAPK pathway (Yamnik and Holz, 2010) (module 7). Inhibition of ESR1 affects activation of $\mathrm{S} 6 \mathrm{~K} 1 / 2$ and stimulates phosphorylation of $\mathrm{S} 6 \mathrm{~K} 1 / 2$ and $4 \mathrm{EBP}$ in the presence of Leucine. ELK1 and ESR1 are regulated by a mTOR MAPK signaling pathway and a VEGF angiogenesis signaling pathway with crosstalk.

SP1 is the most abundant in modules 1 and 2 as well as in other parts of the mTOR pathway. SP1 is a zinc finger protein and binds to motifs rich in GC content in various promoter regions to sustain an open chromatin structure of DNA. SP1 regulates various processes of mTOR pathway, which are cell differentiation, cell growth, apoptosis, immune responses, response to DNA damage, and chromatin remodeling. The allocations of binding motifs for SP1 within promoters of genes in the mTOR pathway suggest the strong possibility of both regulating and regulated statuses of SP1 in the mTOR pathway.

Discovering SP1 in EIF4E1B in module 7 of the mTOR pathway proves the above-mentioned suggestion. EIF4E1B is an inhibitor of eukaryotic translation initiation factor EIF4E-a group of genes involved in mRNA activation, protein synthesis, and cell development ( $\mathrm{Li}$ et al., 2012). Moreover, in the mTOR pathway, SP1 interacts with hypoxia inducible factor HIF1, transactivating vascular endothelial growth factor (VEGF) regulating the VEGF pathway (module 7) and influencing autophagy (module 8) (Alam et al., 2004).

A myeloid-zinc-finger (MZF1) TF function, which is a granulocyte precursor in bone marrow, in the mTOR pathway is not yet discovered. Found in the multiple promoters of module 1 it can be presumed that MZF1 changes the transcription inside the pathway by influencing INS (dependent on growth factors and hormones) or by regulating the MAPK cascade and RPS6K gene (module 1). Moreover, MZF1 is also involved in activating or deactivating the PTEN gene, which is known as a tumor suppressor (module 2). MZF1 binding motifs were also found in VEGFA promoter, suggesting regulation of the VEGF pathway (module 7), in E1F4E1B promoter implying involvement in cell development (module 7), and in ULK1 promoter indicating connection with autophagy process in mTOR (module 8) [27]. Accordingly, MZF1 is a ubiquitous plays an important role in regulation of the whole mTOR pathway.

The third transcription factor contributing largely to the precore modules one and two is PLAG1 (pleiomorphic adenoma gene 1 protein). It regulates a range of mTOR processes involved 
in cell proliferation, organ growth, and apoptosis. In our research, it is found in the promoters of INS, RPS6KA2 (module 1), IRS1, PIK3R1, PIK3CA (module 2), TNF (module 3), and TSC2 (module 4). Akhtar et al. (2012) suggest that PLAG1 is overexpressed in cancer cells when it binds to IGF2 promoter. Because of the close proximity of the IRS1 and IGF genes, PLAG1 may also regulate IRS1 as it was identified in our research.

Pre-core modules 1 (MAPK signaling) and 2 (Insulin signaling) share SP1, MZF1, and PLAG1 transcription factors. Apart from it, module 2 has FOXF2 (forkhead box F2) and TBP (TATAbinding protein). FOXF2 and TBP are already known in the context of co-regulation with MTOR, RDS1, (adenine-repressible gene) and TFAP2A (transcription factor AP-2 alpha) (Westergren, 2010).

FOXF2 is classified as TF, which regulates the mTOR pathway. The increased amount of FOXF2 inhibits the activity of IRS1 (insulin receptor substrate 1 ), leading to inhibition of the whole mTOR pathway via a negative-feedback loop (Westergren, 2010). FOXF2 interacts with another transcription factor TBP (TATAbinding protein), and in our research, both of them were found in close proximity to IRS1. TBP additionally interacts with SP1 and E2F1 transcription factors found in the intersection zone of mostly interconnected genes in our study (Supporting Figure 1A). In module 2, TBP is regulated by $\mathrm{PI} 3 \mathrm{~K}$ genes, and in the mTOR core, TBP is regulated by the MTOR gene, thus, playing a vital role in recruiting Polymerase I and modulating activity of Polymerase III (Cianfrocco et al., 2013).

In consideration of the locations of TFAP2A binding motifs, it is noteworthy that TFAP2A can regulate the mTOR pathway and, in particular, its core path. A TFAP2A binding motif was found in the promoters of IRS1 and PIK3R2 genes of an insulin signaling pathway (module 2), a PTEN gene, which is an inhibitor of the mTOR pathway thought the PIP3 (module 2) and RRAGD genes (module 5). TFAP2A is thus involved in processes of organ development and negative cell proliferation at the same time. One of the probable scenarios concerning TFAP2A is that by inhibition of IRS1, PIK3R2 (module 2), and RRAGD (module 5) genes, TFAP2A stimulates the PTEN transcription immobilizing mTOR pathway and negative feedback loop may exist between insulin-signaling module 2 and amino-acid balance module 5. Surprisingly, a TF characteristic for yeast RDS1 (an adenine-repressible gene), responsible for the regulation of transcription from RNA polymerase II, is identified in the human data set. RDS1 binding motif is found in the promoters of IRS1, AKT1 (threonine-protein kinase) genes of pre-core module 2, and the RRAGC (Ras-related GTP binding C) gene of post-core module 5. It is difficult to assess RDS1 functions in an mTOR pathway due to the fact that it is only been found in yeast to date. However, in yeast organisms, it is responsible for transcription regulation and response to xenobiotic stimulus. In human organisms, its function may be very similar to the function in yeast, and human ortholog might be identified in the future.

RDS1 can influence the mTOR pathway from two sides: first, being regulated by growth factors and hormones (IRS1 and AKT1 in module 2) and, second, being regulated by the availability of amino acids or generally intracellular xenobiotics (RRAGC in module 5). The above-described finding on RDS1 activity indicates the existence of crosstalk between module 2 (insulin signaling) and module 5 (amino-acid balance within a cell) transcription regulation and existence of a negative feedback loop between them. This is a biologically reasonable finding as insulin-control amino-acid metabolism is tightly related to amino-acid-uptake balance.

\section{MODULE FOUR REGULATES HYPOXIA}

ARNT (aryl-hydrocarbon receptor nuclear translocator 3, HIF-1 $\beta$ ) is one that regulates gene expression in hypoxia module 4. It encodes a protein that is crucial for complex formation with the ligand-bound aryl hydrocarbon receptor (AHR) and proper functioning of this receptor. Current knowledge indicates that the HIF1- and/or HIF2-mediated hypoxia responses can be oncogenic as well as tumor suppressive (Pawlis and $\mathrm{Hu}$, 2013). ARNT induces activation of REDD1 (DDIT4) by creating a dimer with HIF1, thereby inhibiting TORC1 and affecting the TSC2-depending mechanism (Kapahi et al., 2010). ARNT interacts with ESR1, co-activating its transcription (Endler et al., 2004), and with SP1, creating a reconstituent complex (Mulero-Navarro et al., 2006) with it. SP1 itself is able to bind the Ah receptor and down-regulates its expression in leukemia cells (Mulero-Navarro et al., 2006).

\section{CORE MODULE OF mTOR}

In the core of mTOR pathway KLF4 (Kruppel-like factor 4) inhibits the MTOR gene and has an anti-proliferative effect on the whole mTOR pathway (Wang et al., 2012), thereby promoting self-renewal and precluding differentiation.

On the contrary, MEF2A (myocyte specific enhancer factor) positively regulates genes of the mTOR/S6K pathway, so it belongs both to the mTOR core and module 7 (VEGF pathway) (Pereira et al., 2009; Yin et al., 2012), promoting cell growth and differentiation. However, the MEF2 family is also responsible for cardiac hypertrophy and failure (Pereira et al., 2009), and its overexpression leads to cardiac dysfunction.

\section{MODULE SEVEN OF VEGF PATHWAY}

Module 7 is associated with VEGF (vascular endothelial growth factor) pathway-advancing cell growth, organ development, and protein formation. This transcriptional module possesses four transcription factors, namely, SP1, ESR1, E2F1, and EGR1, with three first TFs tightly associated with the activities of the mTOR pathway.

EGR1 (early-growth-response protein 1), which is module 7 specific TF, directs cell differentiation. It was discovered that its level in a prostate cancer was raised, showing a pro-oncogenic character; however, in other tumor types (skin tumor, fibrosarcoma, and glioblastoma), EGR1 exhibits features of a tumor suppressor by activating p53 and PTEN to halt the transcription of other genes in the mTOR pathway (Zheng et al., 2009). Moreover, it was discovered that the knockdown of EGR1 affects VEGFA, thereby affecting the VEGF pathway and mitogenesis (Abdel-Malak et al., 2009).

An E2F group affects activation of RPS6KB1 (S6K) and stimulates phosphorylation of $\mathrm{S} 6 \mathrm{~K}$ and $4 \mathrm{EBP}$ in the presence of Leucine. E2F1 integrates cell division (as well as cell growth) and 
induces an apoptotic response (Real et al., 2011). In tumor cells, it can activate EGR1 to endorse cell survival. Moreover, in tumor cells, by up-regulating the production of EGR1, epidermal growth factor (EGFR), platelet-derived growth factor (PDGFRA), and insulin-like growth factor II (IGF2BP2), E2F1 can activate the phosphoinositide-3-kinase/Akt (PIK3CA/AKT) pathway in a way to inhibit drug-induced apoptosis (Zheng et al., 2009).

\section{DISCUSSION}

Transcription factors selected ( 24 genes) from the results analysis where those identified by main and supportive motifs discovery tools used in our study (Supporting Figure 1). Other transcription factors that less frequently appear can be seen in the process-module-assigned fashion in Supporting Figures 1A,B for human and mouse, respectively. Transcription factors (24) are classified into two groups: first, those expressed in the specific module of mTOR and second, those commonly identified in various modules.

The first group of TFs includes ESR1, ELK1 (MAPK signaling, module 1), FOXF2 (Insulin signaling, module 2), KLF4 (mTOR core), ARNT (Hypoxia, module 4), E2F1, and EGR1 (VEGF signaling, module 7). The second group includes SP1, MZF1, PLAG1, TFAP1A, TBP, and RDS1. Among member of the second group, MZF1, PLAG1, TFAP1A, and RDS are newly identified. Other transcription factors identified in the promoters of genes in our analysis are EGR2, EGR3, YY1, SREBF1, SREBF2, ELF1, and ARID3A. They are less frequently share by the promoters in our dataset but they are well-known to be involved in the mTOR pathway regulation (YY1, SREBFs).

One-third (21) of the human transcription factors were found to be conserved in the case of a mouse. Among them most confident are SP1, TFAP2A, MZF1, EGR3, KLF4, USF1, KLF9, and ARNT (Supporting Figure 1B). They are transcription factors having multiple copies in gene promoters and/or shared by many genes within the dataset. Cross-species-conserved TFs identified corroborate the consistency of our methodology. However, a part of frequently appearing mouse TFs, such as HSF1, CDC5L, HES6, and FOXL1, were not identified in orthologous human promoters. This might be regarding to the difference in the transcription regulation between two species.

The results described in the paper indicate that the proper selection of online motif-discovery tools without parameters tuning is feasible to bring accurate results for the discovery transcription regulation on medium size data. However the aim to reduce false positives might result in the omitting low sensitivity-degenerate motifs (Polouliakh et al., 2005). Creation of sophisticated analytic workflow might be warranted to cope with large-scale sequence data for de novo motif discovery.

\section{AUTHOR CONTRIBUTIONS}

Agnieszka Jablonksa analyzed data and wrote the manuscript. Natalia Polouliakh discussed the research and wrote the manuscript.

\section{ACKNOWLEDGMENTS}

We greatly appreciate the scientific support and comments of Prof. Hiroaki Kitano (Sony Computer Science Laboratories Inc.,
Japan), Prof. Huaiyu Mi (University of Southern California, USA) and Dr. Yukiko Matsuoka (Systems Biology Institute, Japan).

\section{SUPPLEMENTARY MATERIAL}

The Supplementary Material for this article can be found online at: http://www.frontiersin.org/journal/10.3389/fcell.2014.00023/ abstract

Supporting Figure 1 | Transcription-factor (TF) binding motifs in the mTOR pathway identified in human (A) and mouse (B) orthologous datasets. The intensity of the blue color reflects frequency of motif occurrence per gene. The colors above transcription-factor names point to those supportive programs (Cscan, LocaMo, TransFind, and TRAP), which also identified a motif after it was detected by the five main programs (MEME_Chip, PASTAA, Pscan, TFM-Explorer, and XXmotif). Transcription factors were sorted according to the sum of occurrences in the whole data set. The red horizontal line in the human-case results highlights 24 genes with average number of motifs per gene higher than 26 (Gene threshold, Gene_TH), and the vertical red line shows the top-24 transcription factors (TF_TH) identified as the most significant in our study. Sub-table (B) shows mouse transcription factors and genes in the same order with the human ones and extended red lines from human data in order to depict similarities between two species. Sub-table (C) includes description of transcription factors and estimated positions of their motifs in human promoters based on the results of the five main programs (MEME_Chip, PASTAA, Pscan, TFM-Explorer, and XXmotif) and asterisks point to human-mouse orthologous TFs.

\section{REFERENCES}

Abdel-Malak, N. A., Mofarrahi, M., Mayaki, D., Khachigian, L. M., and Hussain, S. N. A. (2009). Early growth response-1 regulates angiopoietin-1-induced endothelial cell proliferation, migration, and differentiation. Arterioscler. Thromb. Vasc. Biol. 29, 209-216. doi: 10.1161/ATVBAHA.108.181073

Akhtar, M., Holmgren, C., Göndör, A., Vesterlund, M., Kanduri, C., Larsson, C., et al. (2012). Cell type and context-specific function of PLAG1 for IGF2 P3 promoter activity. Int. J. Oncol. 41, 1959-1966. doi: 10.3892/ijo.2012.1641

Alam, H., Maizels, E. T., Park, Y., Ghaey, S., Feiger, Z. J., Chandel, N. S., et al. (2004). Follicle-stimulating Hormone Activation of Hypoxia-inducible Factor1 by the Phosphatidylinositol 3-Kinase/AKT/Ras Homolog Enriched in Brain (Rheb)/Mammalian Target of Rapamycin. J. Biol. Chem. 279, 19431-19440. doi: 10.1074/jbc.M401235200

Ao, W., Gaudet, J., Kent, W. J., Muttumu, S., and Mango, S. E. (2004). Environmentally induced foregut remodeling by PHA-4/FoxA and DAF12/NHR. Science 305, 1743-1746. doi: 10.1126/science.1102216

Bailey, T. L., Boden, M., Buske, F. A., Frith, M., Grant, C. E., Clementi, L., et al. (2009). MEME SUITE: tools for motif discovery and searching. Nucleic Acids Res. 37, W202-W208. doi: 10.1093/nar/gkp335

Blagosklonny, M. V. (2006). Aging and Immortality Quasi-programmed Senescence and Its Pharmacologic Inhibition. Cell Cycle 5, 2087-2102. doi: 10.4161/cc.5.18.3288

Broos, S., Hulpiau, P., Galle, J., Hooghe, B., Roy, V. F., and Bleser, P. D. (2011). ConTra v2: a tool to identify transcription factor binding sites across species, update 2011. Nucleic Acids Res. 39, W74-W78. doi: 10.1093/nar/gkr355

Brown, E. J., Albers, M. W., Shin, T. B., Ichikawa, K., Keith, C. T., Lane, W. S., et al. (1994). A mammalian protein targeted by G1-arresting rapamycin-receptor complex. Nature 369, 756-758. doi: 10.1038/369756a0

Carlson, J. M., Chakravarty, A., DeZiel, C. E., and Gross, R. H. (2007). SCOPE: a web server for practical de novo motif discovery. Nucleic Acids Res. 35, W259-W264. doi: 10.1093/nar/gkm310

Caron, E., Ghosh, S., Matsuoka, Y., Ashton-Beaucage, D., Therrien, M., Lemieux, L., et al. (2010). A comprehensive map of the mTOR signaling network. Mol. Syst. Biol. 6, 453. doi: 10.1038/msb.2010.108

Chaivorapol, C., Melton, C., Wei, G., Yeh, R. F., Ramalho-Santos, M., Blelloch, R., et al. (2008). CompMoby: comparative MobyDick for detection of cisregulatory motifs. BMC Bioinformatics 9:455. doi: 10.1186/1471-2105-9-455 
Cianfrocco, M. A., Kassavetis, G. A., Grob, P., Fang, J., Juven-Gershon, T., Kadonaga, J. T., et al. (2013). Human TFIID binds to core promoter DNA in a reorganized structural state. Cell 152, 120-131. doi: 10.1016/j.cell.2012.12.005

Defrance, M., Touzet, H. (2006). Predicting transcription factor binding sites using local over-representation and comparative genomics BMC Bioinformatics 7:396. doi: 10.1186/1471-2105-7-396

Deprost, D., Yao, L., Sormani, R., Moreau, M., Leterreux, G., Nicola, M., et al. (2007). The Arabidopsis TOR kinase links plant growth, yield, stress resistance and mRNA translation. EMBO Rep. 8, 864-870. doi: 10.1038/sj.embor. 7401043

Dinh, H., Rajasekaran, S., and Kundeti, V. K. (2011). PMS5: an efficient exact algorithm for the (1,d)-motif finding problem. BMC Bioinformatics 12:410. doi: 10.1186/1471-2105-12-410

Endler, A., Chen, L., Zhang, J., Xu, G. T., and Shibasaki, F. (2004). Binding of the ER_and ARNT1 AF2 domains to exon 21 of the SRC1 isoform SRC1e is essential for estrogen- and dioxin-related transcription. J. Cell Sci. 125, 2004-2016 doi: 10.1242/jcs.097246

Favorov, A. V., Gelfand, M. S., Gerasimova, A. V., Ravcheev, D. A., Mironov, A. A., and Makeev, V. J. (2005). A Gibbs sampler for identification of symmetrically structured, spaced DNA motifs with improved estimation of the signal length. Bioinformatics 21:10. doi: 10.1093/bioinformatics/bti336

Fu, Y., Frith, M. C., Haverty, P. M., and Weng, Z. (2004). MotifViz: an analysis and visualization tool for motif discovery. Nucleic Acids Res. 32, W420-W423. doi: 10.1093/nar/gkh426

Funahashi, A., Tanimura, N., Morohashi, M., and Kitano, H. (2003). CellDesigner: a process diagram editor for gene-regulatory and biochemical networks. Biosilico 1, 159-162. doi: 10.1016/S1478-5382(03)02370-9

Ghosh, S., Matsuoka, Y., Asai, Y., Hsin, K. Y., and Kitano, H. (2011). Software for systems biology: from tools to integrated platforms. Nat. Rev. Genet. 12, 821-832. doi: 10.1038/nrg3096

Hall, M. N. (2008). mTOR-what does it do? Transplant. Proc. 40, S5-S8. doi: 10.1016/j.transproceed.2008.10.009

Hay, N., and Sonenberg, N. (2004). Upstream and downstream of mTOR. Genes Dev. 18, 1926-1945. doi: 10.1101/gad.1212704

Heinemeyer, T., Wingender, E., Reuter, I., Hermjakob, H., Kel, A. E., Kel, O. V., et al. (1998). Databases on transcriptional regulation: TRANSFAC, TRRD and COMPEL. Nucleic Acids Res. 26, 362-367. doi: 10.1093/nar/ 26.1.362

Heitman, J., Movva, N. R., and Hall, M. N. (1991). Targets for cell cycle arrest by the immunosuppressant rapamycin in yeast. Science 253, 905-909. doi: $10.1126 /$ science. 1715094

Inoki, K., and Guan, K. L. (2006). Complexity of the TOR signaling network. Trends Cell Biol. 16, 206-212. doi: 10.1016/j.tcb.2006.02.002

Inoki, K., Ouyang, H., Li, Y., and Guan, K. L. (2005). Signaling by target of rapamycin proteins in cell growth control. Microbiol. Mol. Biol. Rev. 69, 79-100. doi: 10.1128/MMBR.69.1.79-100.2005

Johnson, S. C., Rabinovitch, P. S., and Kaeberlein, M. (2013). mTOR is a key modulator of ageing and age-related diseases. Nature 493, 338. doi: 10.1038/nature11861

Kapahi, P., Chen, D., Rogers, A. N., Katewa, S. D., Li, P. W., Thomas, E. L., et al. (2010). With TOR, less is more: a key role for the conserved nutrient-sensing TOR pathway in aging. Cell Metab. 11, 453-65. doi: 10.1016/j.cmet.2010. 05.001

Kielbasa, S. M., Klein, H., Roider, H. G., Vingron, M., and Blüthgen, N. (2010). TransFind - predicting transcriptional regulators for gene sets. Nucleic Acids Res. 38, W275-W280. doi: 10.1093/nar/gkq438

Kobayashi, T., Minowa, O., Sugitani, Y., Takai, S., Mitani, H., Kobayashi, E., et al. (2001). A germ-line Tscl mutation causes tumor development and embryonic lethality that are similar, but not identical to, those caused by Tsc 2 mutation in mice. Proc. Natl. Acad. Sci. U.S.A. 98, 8762-8769. doi: 10.1073/pnas.151033798

Laplante, M., and Sabatini, D. M. (2009). mTOR signaling at a glance. J. Cell Sci. 122, 3589-3594. doi: 10.1242/jcs.051011

Lee, C., and Huang, C. H. (2013). LASAGNA-Search: an integrated web tool for transcription factor binding site search and visualization. BioTechniques 54, 141-153. doi: 10.2144/000113999

Li, N., Zhong, M., and Song, M. (2012). Expression of phosphorylated mTOR and its regulatory protein is related to biological behaviors of ameloblastoma. Int. J. Clin. Exp. Pathol. 5, 660-667.
Liu, X., Brutlag, D. L., and Liu, J. S. (2001). Bioprospector: discovering conserved dna motifs in upstream regulatory regions of co-expressed genes. Pac. Symp. Biocomput. 6, 127-138.

Liu, X. S., Brutlag, D. L., and Liu, J. S. (2002). An algorithm for finding proteinDNA binding sites with applications to chromatin-immunoprecipitation microarray experiments. Nat. Biotechnol. 8, 835-839. doi: 10.1038/ nbt717

Liu, Y., Liu, X. S., Wei, L., Altman, R. B., and Batzoglou, S. (2004). Eukaryotic regulatory element conservation analysis and identification using comparative genomics. Genome Res. 14, 451-458 doi: 10.1101/gr.1327604

Loots, G. G., and Ovcharenko, I. (2004). rVISTA 2.0: evolutionary analysis of transcription factor binding sites. Nucleic Acids Res. 32, W217-W221. doi: 10.1093/nar/gkh383

Lorberg, A., and Hall, M. N. (2004). TOR: the first 10 years. Curr. Top Microbiol. Immunol. 279, 1-18. doi: 10.1007/978-3-642-18930-2_1

Luehr, S., Hartmann, H., and Söding, J. (2012). The XXmotif web server for exhaustive, weight matrix-based motif discovery in nucleotide sequences. Nucleic Acids Res. 40, W104-W109. doi: 10.1093/nar/gks602

Mulero-Navarro, S., Carvajal-Gonzalez, J. M., Herranz, M., Ballestar, E., Fraga, M. F., Ropero, S., et al. (2006). The dioxin receptor is silenced by promoter hypermethylation in human acute lymphoblastic leukemia through inhibition of Sp1 binding. Carcinogenesis 27, 1099-1104. doi: 10.1093/carcin/bgi344

Murakami, M., Ichisaka, T., Maeda, M., Oshiro, N., Hara, K., Edenhofer, F., et al. (2004). mTOR is essential for growth and proliferation in early mouse embryos and embryonic stem cells. Mol. Cell. Biol. 24, 6710-6718. doi: 10.1128/MCB.24.15.6710-6718.2004

Néron, B., Ménager, H., Maufrais, C., Joly, N., Maupetit, J., Letort, S., et al. (2009). Mobyle: a new full web bioinformatics framework. Bioinformatics 25, 3005-3011. doi: 10.1093/bioinformatics/btp493

O'Neill, L. A. J., and Hardie, D. G. (2013). Metabolism of inflammation limited by AMPK and pseudo-starvation. Nature 493, 346-355.

Pawlis, M. R., and Hu, C. J. (2013). Enhanceosomes as integrators of hypoxia inducible factor (HIF) and other transcription factors in the hypoxic transcriptional response. Cell. Signal. 25, 1895-903. doi: 10.1016/j.cellsig.2013. 05.018

Pereira, A. H., Clemente, C. F., Cardoso, A. C., Theizen, T. H., Rocco, S. A., Judice, C. C., et al. (2009). MEF2C silencing attenuates load-induced left ventricular hypertrophy by modulating mTOR/S6K pathway in mice. PLoS ONE 4:e8472. doi: 10.1371/journal.pone.0008472

Machanick, P, and Bailey, T. L. (2011). MEME-ChIP: motif analysis of large DNA datasets. Bioinformatics 27, 1696-1697. doi: 10.1093/bioinformatics/btr189

Polouliakh, N., Konno, M., Horton, P., and Nakai, K. (2005). Parameter lanscape analysis for common motif discovery programs. Lect. Notes Comput. Sci. 3318, 79-87. doi: 10.1007/978-3-540-32280-1_8

Polouliakh, N., Natsume, T., Harada, H., Fujibuchi, W., and Horton, P. (2006). Comparative genomic analysis of transcription regulation elements involved in human map kinase G-protein coupling pathway. J. Bioinform. Comput. Biol. 4, 469-482. doi: 10.1142/S0219720006001849

Polouliakh, N., Nock, R., Nielsen, F., and Kitano, H. (2009) G-Protein coupled receptor signaling architecture of mammalian immune cells. PLoS ONE 4:e4189. doi: 10.1371/journal.pone.0004189

Poluliakh, N., Takagi, T., and Nakai, K. (2003). MELINA: motif extraction from promoter regions of potentially co-regulated genes. Bioinformatics 19, 423-424. doi: 10.1093/bioinformatics/btf872

Real, S., Meo-Evoli, N., Espada, L., and Tauler, A. (2011). E2F1 regulates cellular growth by mTORC1 signaling. PLOS ONE 6:e16163. doi: 10.1371/journal.pone.0016163

Roider, H. G., Kanhere, A., Manke, T., and Vingron, M. (2007). Predicting transcription factor affinities to DNA from a biophysical model. Bioinformatics 23, 134-141. doi: 10.1093/bioinformatics/btl565

Roider, H. G., Manke, T., O'Keeffe, S., Vingron, M., and Haas, S. A. (2009). PASTAA: identifying transcription factors associated with sets of co-regulated genes. Bioinformatics 25, 435-442. doi: 10.1093/bioinformatics/ btn627

Romer, K. A., Kayombya, G. R., and Fraenkel, E. (2007). WebMOTIFS: automated discovery, filtering and scoring of DNA sequence motifs using multiple programs and Bayesian approaches. Nucleic Acids Res. 35, W217-W220. doi: $10.1093 /$ nar/gkm376 
Sabatini, D. M., Erdjument-Bromage, H., Lui, M., Tempst, P., and Snyder, S. H. (1994). RAFT1: a mammalian protein that binds to FKBP12 in a rapamycindependent fashion and is homologous to yeast TORs. Cell 78, 35-43. doi: 10.1016/0092-8674(94)90570-3

Sandelin, A., Wasserman, W. W., and Lenhard, B. (2004). ConSite: web-based prediction of regulatory elements using cross-species comparison. Nucleic Acids Res. 32, W249-W252. doi: 10.1093/nar/gkh372

Schmelzle, T., and Hall, M. N. (2000). TOR, a central controller of cell growth. Cell 103, 253-262. doi: 10.1016/S0092-8674(00)00117-3

Serizawa, S., Miyamichi, K., Nakatani, H., Suzuki, M., Saito, M., Yoshihara, Y., et al. (2003). Negative feedback regulation ensures the one receptor - one olfactory neuron rule in mouse. Science 19, 2088-2094 doi: 10.1126/science. 1089122

Siddharthan, R., Siggia, E. D., and van Nimwegen, E. (2005). PhyloGibbs: a Gibbs sampling motif finder that incorporates phylogeny. PLoS Comput. Biol. 1:e67. doi: 10.1371/journal.pcbi.0010067

Sinha, S., and Tompa, M. (2003). YMF: a program for discovery of novel transcription factor binding sites by statistical overrepresentation. Nucleic Acids Res. 31, 3586-3588. doi: 10.1093/nar/gkg618

Stanfel, M. N., Shamieh, L. S., Kaeberlein, M., and Kennedy, B. K. (2009). The TOR pathway comes of age. Biochim. Biophys. Acta 1790, 1067-1074. doi: 10.1016/j.bbagen.2009.06.007

Thompson, W., Rouchka, E. C., and Lawrence, C. E. (2003). Gibbs Recursive Sampler: finding transcription factor binding sites. Nucleic Acids Res. 31, 3580-3585. doi: 10.1093/nar/gkg608

Uht, R. M., Amos, S., Martin, P. M., Riggan, A. E., and Hussaini, I. M. (2007). The protein kinase C-g isoform induces proliferation in glioblastoma cell lines through an ERK/Elk-1 pathway. Oncogene 30, 2885-2893. doi: 10.1038/sj.onc. 1210090

Vandenbon, A., Kumagai, Y., Teraguch, S., Amada, K. M., Akira, S., Daron, M., et al. (2013). A Parzen window-based approach for the detection of locally enriched transcription factor binding sites. BMC Bioinformatics 14:26. doi: 10.1186/14712105-14-26

Wang, Y., Zhao, B., Zhang, Y., Tang, Z., Shen, Q., Zhang, Y., et al. (2012). Krüppellike factor 4 is induced by rapamycin and mediates the anti-proliferative rapamycin in rat carotid arteries. Br. J. Pharmacol. 165, 2378-2388. doi: 10.1111/j.1476-5381.2011.01734.x

Westergren, R. (2010). Foxf2 and Foxc2, Two Transcription Factors that Regulate Adipocyte Metabolism. Gothenburg: Intellecta Infolog.

Wijaya, E., Yiu, S. M., Son, N. T., Kanagasabai, R., and Sung, W. K. (2008). MotifVoter: a novel ensemble method for fine-grained integration of generic motif finders. Bioinformatics 24, 2288-2295. doi: 10.1093/bioinformatics/btn 420

Yamashita, R., Wakaguri, H., Sugano, S., Suzuki, Y., and Nakai K. (2010). DBTSS provides a tissue specific dynamic view of Transcription Start Sites. Nucleic Acids Res. 38, D98-D104. doi: 10.1093/nar/gkp1017

Yamnik, R. L., and Holz, M. K. (2010). mTOR/S6K1 and MAPK/RSK signaling pathways coordinately regulate estrogen receptor a serine 167 phosphorylation. FEBS Lett. 584, 124-128. doi: 10.1016/j.febslet.2009.11.041

Yin, Y., She, H., Li, W., Yang, Q., Guo, S., and Mao, Z. (2012). Modulation of neuronal survival factor MEF2 by kinases in Parkinson's disease. Front. Physiol. 3:171. doi: 10.3389/fphys.2012.00171

Zambelli, F., Pesole, G., and Pavesi, G. (2009). Pscan: finding over-represented transcription factor binding site motifs in sequences from co-regulated or co-expressed genes. Nucleic Acids Res. 37, W247-W252. doi: 10.1093/nar/ gkp464

Zambelli, F., Prazzoli, G. M., Pesole, G., and Pavesi, G. (2012). Cscan: finding common regulators of a set of genes by using a collection of genomewide ChIP-seq datasets. Nucleic Acids Res 40, W510-W51. doi: 10.1093/nar/ gks483

Zheng, C., Ren, Z., Wang, H., Zhang, W., Kalvakolanu, D. V., Tian, Z., et al. (2009). E2F1 Induces tumor cell survival via nuclear factor-kappa B-dependent induction of EGR1 transcription in prostate cancer cells. Cancer Res. 69, 2324-2331. doi: 10.1158/0008-5472.CAN-08-4113

Conflict of Interest Statement: The authors declare that the research was conducted in the absence of any commercial or financial relationships that could be construed as a potential conflict of interest.

Received: 01 April 2014; paper pending published: 13 April 2014; accepted: 09 May 2014; published online: 03 June 2014.

Citation: Jablonska A and Polouliakh N (2014) In silico discovery of novel transcription factors regulated by mTOR-pathway activities. Front. Cell Dev. Biol. 2:23. doi: $10.3389 /$ fcell.2014.00023

This article was submitted to Systems Biology, a section of the journal Frontiers in Cell and Developmental Biology.

Copyright (C) 2014 Jablonska and Polouliakh. This is an open-access article distributed under the terms of the Creative Commons Attribution License (CC BY). The use, distribution or reproduction in other forums is permitted, provided the original author(s) or licensor are credited and that the original publication in this journal is cited, in accordance with accepted academic practice. No use, distribution or reproduction is permitted which does not comply with these terms. 